\title{
A Comparison of Centering Algorithms in the Astrometry of Cassini Imaging Science Subsystem Images and Anthe's Astrometric Reduction
}

\author{
Q. F. Zhang, ${ }^{1,2}$ X. M. Zhou, ${ }^{1}$ Y. Tan,${ }^{1}$ V. Lainey, ${ }^{3 \star}$ N. J. Cooper,${ }^{4} \dagger$ A. Vienne, ${ }^{3}$ \\ W. H. Qin, ${ }^{1}$ Z. Li $^{1,2}$ and Q. Y. Peng ${ }^{1,2}$ \\ ${ }^{1}$ Department of Computer Science, Jinan University, Guangzhou 510632, P. R. China \\ ${ }^{2}$ Sino-French Joint Laboratory for Astrometry, Dynamics and Space Science, Jinan University, Guangzhou, 510632, P. R. China \\ ${ }^{3}$ IMCCE, Observatoire de Paris, UMR 8028 du CNRS, UPMC, Universite de Lille 1, 77 av. Denfert-Rochereau, 75014 Paris, France \\ ${ }^{4}$ Astronomy Unit, School of Physics $\mathcal{E}$ Astronomy, Queen Mary University of London, Mile End Road, London E1 4NS, UK
}

Accepted XXX. Received YYY; in original form ZZZ

\begin{abstract}
In the Caviar software package, a standard tool for astrometry of images from the Cassini Imaging Science Subsystem (ISS), Gaussian fitting is used to measure the centre of pointlike objects, achieving a typical precision of about 0.2 pixels. In this work, we consider how alternative methods may improve on this. We compare three traditional centroiding methods: two-dimensional Gaussian fitting, median, and modified moment. Results using 56 selected images show that the centroiding precision of the modified moment method is significantly better than the other two methods, with standard deviations for all residuals in sample and line of 0.065 and 0.063 pixels, respectively, representing a factor of over 2 improvement compared to Gaussian fitting. Secondly, a comparison of observations using Cassini ISS images of Anthe is performed. Anthe results show a similar improvement. The modified moment method is then used to reduce all ISS images of Anthe during the period 2008-2017. The observedminus-calculated residuals relative to the JPL SAT393 ephemeris are calculated. In terms of $\alpha \times \cos (\delta)$ and $\delta$ in the Cassini-centred international celestial reference frame, mean values of all residuals are close to $0 \mathrm{~km}$, and their standard deviations are less than $1 \mathrm{~km}$ for narrow angle camera images, and about $4 \mathrm{~km}$ for wide angle camera images.
\end{abstract}

Key words: astrometry - ephemerides - planets and satellites: individual: Anthe - techniques: image processing-methods: observational

\section{INTRODUCTION}

The Cassini orbiter carried an optical Imaging Science Subsystem (ISS) (Porco et al. 2004) which recorded more than 440,000 images during the course of the mission. Both during, and after the end of the mission, these images have and continue to be an important resource for natural satellite astrometry. For example, Cooper et al. (2006) reduced ISS images of inner Jovian satellites, while Cooper et al. (2014) performed mutual-event astrometry of ISS images of the mid-sized Saturnian satellites and Tajeddine et al. (2013, 2015) and Zhang et al. (2018) reduced images of the main icy Saturnian satellites. A software package, Caviar $^{1}$, dedicated to the astromet-

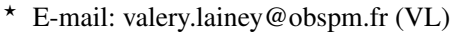

$\dagger$ E-mail: n.cooper@qmul.ac.uk (NJC)

E-mail: tpengqy@jnu.edu.cn (QYP)

1 Available under Creative Commons Attribution-NonCommercialShareAlike 4.0 International License (https://www.imcce.fr/recherche /equipes/pegase/caviar)
}

ric reduction of Cassini ISS images has since been released to the community (Cooper et al. 2018). The importance and value of this high-precision astrometric dataset have recently been demonstrated by Lainey et al. (2020), who combined it with Cassini radio science data to show that the Saturnian moon Titan has been migrating away from Saturn on a timescale of roughly ten billion years, implying that Saturn is an order of magnitude more tidally dissipative than previously thought.

A key step in the astrometric reduction of Cassini ISS images is the use of star positions, measured using a centroiding technique, to correct the nominal camera pointing direction for each image. Besides, if the observed target satellite's image is also point-like, its photocentre is also measured using a centroiding technique. So centroiding in general plays an important role in the astrometry of ISS images.

Various centroiding methods have already been described in the literature. Stone (1989) used synthetically produced star images to compare the speed, convergence, and processing accuracy of some classical centroiding algorithms, such as moment, mod- 
ified moment, Gaussian fitting, median, and a derivative search method, concluding that the modified moment method has certain advantages over the other methods when the sky-background noise level is significant. But no real images were used to evaluate these methods. Anderson et al. (2000) presented an effective Point Spread Function (ePSF) method, which is an excellent highprecision method and significantly improves the astrometric accuracy of stars in HST images. However, many stars (tens or even hundreds) in one image are required to build an accurate ePSF model. In addition, the ePSF model varies with the observation conditions. These two issues restrict the application of the ePSF method. Delabie et al. (2014) proposed a Gaussian grid algorithm that could quickly and accurately calculate the position of stars in an image. As described in that paper, its accuracy is worse than that of the two-dimensional Gaussian fitting method, although it has high efficiency. Sun \& Zhao (2014) used the modified moment method to calculate the centroid of space debris and offered an adaptive scheme to provide the threshold used in measurements. The result showed that this method improved the astrometric precision for space debris centroiding. However, it is not generally suitable for the astrometry of ISS images because in these images, stars are point-like, instead of the streaked images typical of space debris. Wang et al. (2015) developed a Gaussian analytical centroiding method for star trackers, which featured a pure analytical form, better precision and high speed. But this method was limited by the assumption that the simulated reference star image followed a Gaussian law, which is not always the case. Lu et al. (2018) proposed a centroiding algorithm based on Fourier spatial phase fitting, and tested it in Galsim and CFHT (Canada France Hawaii Telescope)-like simulated star images, demonstrating that it had better accuracy than the Gaussian method provided that the stars' images were sufficiently well-sampled, which again, is often not the case.

The previous work in this area, summarised above, demonstrates how centroiding methods may lead to a differing performance in different situations of application. Cassini ISS images have their own particular characteristics. For example, reference stars are generally below 15 th magnitude. Few are brighter than 8 th magnitude, while most are around 9th or 10th magnitude. Star images are also typically sharp, and non-Gaussian, often consisting of one or two pixels only. In the Caviar software package (Cooper et al. 2018), two-dimensional Gaussian fitting is currently used. However, it is not clear that this method performs optimally for Cassini ISS images and the motivation of this paper is to compare it with other available methods.

The small inner Saturnian satellite Anthe (S/2007 S 4), first discovered using Cassini ISS images (Porco et al. 2007; Cooper et al. 2008), orbits in the region between Methone and Pallene, and has since been the subject of considerable further interest. Hedman et al. (2008); Madeira et al. (2020) studied the resonant association between Anthe, Methone and Pallene, and arcs of dusty material. Sun et al. (2017) modelled the source, dynamical evolution, and sinks of the dust contained in the arcs of material associated with the orbits of Methone and Anthe. Munoz-Gutierrez et al. (2017) studied the long-term dynamical evolution and stability of four small Saturnian satellites, including Anthe. In terms of the existing published astrometry of Anthe, at the time of its discovery, Cooper et al. (2008) reduced 63 Cassini ISS images taken between 2004 and 2007. In this paper, we will use a high-precision method to reduce all the remaining ISS images of Anthe taken between 2008 and the end of the Cassini mission, in 2017.

We introduce the principles of the three classical centroiding

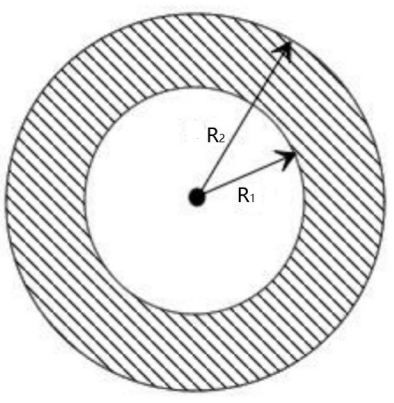

Figure 1. Determination of the background region of a star image.

algorithms in Section 2. In Section 3, we describe the comparative experiment for the three methods, using a selection of ISS images, and analyze the results to select the best centroiding method overall. In Section 4, we use the modified moment method to measure Narrow Angle Camera (NAC) images of Anthe, and compare the results with the Gaussian fitting method. Then, the complete set of NAC and Wide Angle Camera (WAC) images of Anthe from 2008-2017 are reduced by the modified moment method. Finally, we summarise and conclude in Section 5.

\section{CLASSICAL CENTRING ALGORITHMS}

Many existing centroiding algorithms are based on implementations of either the modified moment, two-dimensional Gaussian fitting or median methods, and these have generally been shown to be simple and efficient to use. Their basic principles are briefly introduced below. See also Stone (1989) for more details.

\subsection{Modified moment}

Given one star image $I(x, y)$. The star's centroid is $\left(x_{0}, y_{0}\right)$. Modified moment takes the centroid as the first moment of the intensity distribution in the star image. Its basic formula is as follows:

$$
\begin{gathered}
x_{0}=\left(\sum_{x=x_{\text {min }}}^{x_{\max }} \sum_{y=y_{\min }}^{y_{\max }} x I^{\prime}(x, y)\right) / A \\
y_{0}=\left(\sum_{x=x_{\min }}^{x_{\max }} \sum_{y=y_{\min }}^{y_{\max }} y I^{\prime}(x, y)\right) / A . \\
A=\sum_{x=x_{\min }}^{x_{\max }} \sum_{y=y_{\min }}^{y_{\max }} I^{\prime}(x, y)
\end{gathered}
$$

Where $I^{\prime}(x, y)$ is the modified intensity of the pixel at coordinates $(x, y)$. The constants $x_{\min }, x_{\max }, y_{\min }$ and $y_{\max }$ define the left, right, bottom and top of the target box containing the star in the image, respectively. In practice, the target box is often square. $A$ is the total modified intensity of the target box. $I^{\prime}(x, y)$ is obtained by the equations:

$I^{\prime}(x, y)= \begin{cases}I(x, y)-T & I(x, y)>T \\ 0 & I(x, y) \leqslant T .\end{cases}$

$T=b+3 \sigma$.

Where, $I(x, y)$ is the original intensity at coordinates $(x, y) . T$ is a threshold. The average and standard deviation of the star's background intensity are $b$ and $\sigma$, respectively. In this method, $T$ plays the role of mitigating the effects of noise in centroiding. The chosen value of $T$ should be a trade-off between removing noise while not adversely affecting the star's signal. In general, $T$ is chosen at a $2 \sigma \sim 3 \sigma$ level above that of the background. For high-precision 
astrometry in particular, it should be handled carefully. Here, we chose a value at the $3 \sigma$ level, following experiments.

From eq. (3), we know that getting a reasonable value of $T$ depends on locating an accurate background level. Here, to find the background level, we developed a scheme that was applied on Cassini ISS images. For point-like star images, we assume that the background area is an annular area as in Fig. 1. In the figure, the black dot is the star's center $\left(x_{0}, y_{0}\right)$, the shaded region is its background. The shaded annular area is determined by the values of $R_{1}$ and $R_{2}$. We follow the steps below to estimate these values:

(1) Set the centroid coordinates of the star to be an initial value $\left(x_{0}, y_{0}\right)$, as obtained by the chosen star search algorithm. Typically, the accuracy of this initial position estimate is $\frac{1}{3}$ to $\frac{1}{4}$ pixel (Tajeddine et al. 2013). We assume that the error between the initial centroid and the true one is less than 1.5 pixels both in $\mathrm{x}$ and $\mathrm{y}$ direction.

(2) Modify the initial centroid: look for the pixel with peak grey value in the $3 \times 3$ neighbourhood centred at the initial centroid, and replace the initial centroid with the peak's location. If the peak cannot be found, preserve the initial centroid.

(3) Considering that the full-width at half-maximum (FWHM) of the point spread function for the ISS NAC is 1.3 pixels (Porco et al. 2004), we set $R_{0}=0$ pixel, $R_{1}=1$ pixel, $R_{2}=2$ pixels.

(4) Calculate the mean intensity $M_{i n}$ in the annular area with inner radius $R_{0}$ and outer radius $R_{1}$, and the mean intensity $M_{\text {out }}$ in the annular area with inner radius $R_{1}$ and outer radius $R_{2}$.

(5) Calculate the ratio $=M_{\text {in }} / M_{\text {out }}$.

(6) If $M_{\text {in }}<25$ or ratio $\leq 1.01$, the annular area with the inner radius $R_{1}=R_{2}$ and outer radius $R_{2}=R_{2}+6$ is the background area. Compute the value of $b$ and $\sigma$ of the background. Stop the procedure.

(7) Else, set $R_{0}=R_{0}+1, R_{1}=R_{1}+1, R_{2}=R_{2}+1$ and repeat steps (4) - (7).

\subsection{Two-dimensional Gaussian fitting}

As described in the modified moment method, given one star image $I(x, y)$, the star's centroid is $\left(x_{0}, y_{0}\right)$. According to the twodimensional Gaussian fitting method, the intensity distribution of the star image satisfies the following equation:

$G(x, y)=b+H \exp \left(-\frac{\left(x-x_{0}\right)^{2}+\left(y-y_{0}\right)^{2}}{2(\sigma)^{2}}\right)$.

Where $b$ is sky background. $\mathrm{H}$ is the central image height in the distribution. $\sigma$ is the root-mean-squared half-width of the star image. A Gaussian function is used to fit the intensity distribution in the target box, and the centre of the Gaussian function is taken to be the required centre of the star. In this work, 2D Gaussian fitting was performed using the IDL application of the DAOPHOT find technique (Stetson 1987).

\subsection{Median}

In the median method, the point bisecting the cumulative distribution function of the target star is taken as its centre. Firstly, a one-dimensional distribution curve of the target in the $\mathrm{X}$ direction is calculated according to:

$Q(X)=\sum_{x=x_{\min }}^{X} \sum_{y=y_{\min }}^{y_{\max }} I^{\prime}(x, y)$.

Where, the variable $X \in\left[x_{\min }, x_{\max }\right] . I^{\prime}(x, y), x_{\min }, x_{\max }, y_{\min }$

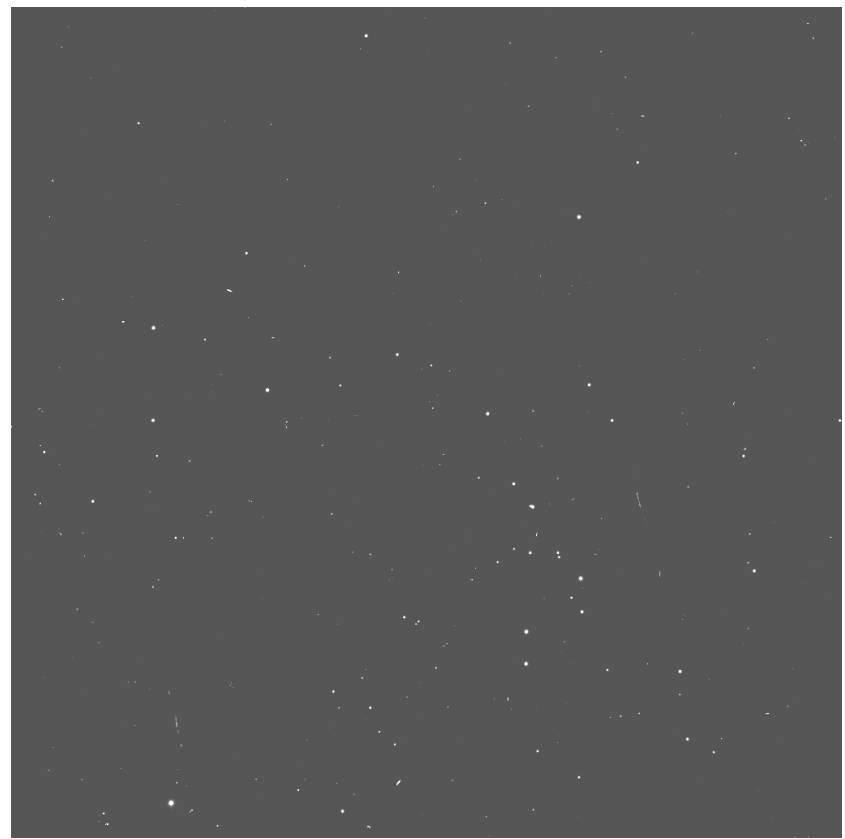

Figure 2. Example of an ISS image, image name: N1601335746. This image was changed by $\log$ transformation for more visibility.

and $y_{\max }$ are as defined in the modified moment method. $Q(X)$ is the cumulative intensity distribution function along the $X$ direction. The median method involves finding $X_{0}$ on the curve $X-Q(X)$ that satisfies the expression:

$Q\left(X_{0}\right)=\frac{1}{2}\left(Q\left(x_{\max }\right)-Q\left(x_{\min }\right)\right)$.

To get an accurate $X_{0}$, interpolation must be used to fit the curve $X-Q(X)$ in a given interval. Generally, cubic interpolation is applied to obtain $X_{0}$. The process in the $\mathrm{Y}$ direction is the same as that in X.

In our implementation, we use the same scheme in section 2.1 to compute the modified intensity $I^{\prime}(x, y)$.

\section{COMPARISON BETWEEN THE THREE METHODS}

To evaluate the three centroiding algorithms for use in the astrometry of ISS images, the following procedure was used: (1) select some suitable ISS images; (2) use different centroiding algorithms to measure the centre position of each star in these images; (3) compare these measured positions with their corresponding reduced positions from the Gaia DR2 catalogue (Gaia Collaboration 2018a,b) to obtain their residuals; (4) compare the accuracy and precision of all residuals from each method.

Firstly, 56 images (image series N1533083910 to N1533095430 and N1601334486 to N1601342286) were selected. Each of these images was targeted at sky (not satellite or planet), so that there are only stars in an image. This eliminates any possible disturbance from solar system objects, and provides a proper distribution of stars in one image to benefit the positional measurement of each star. Each image had about 110 detectable stars, within the image size of $1024 \times 1024$ pixels. Each was taken with filters CL1 and CL2 and exposure time of 2.6 seconds. Fig. 2 shows a representative image, in which the stars' magnitudes range from 7.8 to 14. Fig. 3 shows some local star images with different 

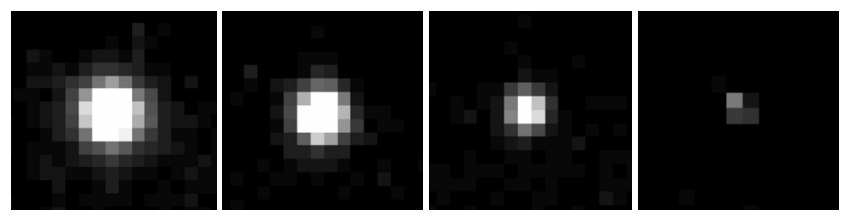

Figure 3. Local images of stars with different magnitudes. From left to right, their magnitudes are 8.8, 9.6, 11.0, 13.5, respectively. All images were magnified for more visibility.

Table 1. Mean values (mean) and standard deviations (std) of residuals of all 3845 observed positions relative to Gaia DR2 in sample and line, unit in pixels.

\begin{tabular}{c|c|cc|cc|cc}
\hline \multirow{2}{*}{ magnitude } & \multirow{2}{*}{ coordinate } & \multicolumn{2}{|c|}{ Gaussian } & \multicolumn{2}{c|}{ Median } & \multicolumn{2}{c}{ Modified Moment } \\
\cline { 3 - 8 } & & mean & std & mean & std & mean & std \\
\hline \multirow{2}{*}{$8-9$} & sample & -0.005 & 0.132 & -0.009 & 0.095 & -0.006 & 0.044 \\
& line & -0.038 & 0.116 & -0.017 & 0.094 & -0.021 & 0.049 \\
\hline \multirow{2}{*}{$9-10$} & sample & -0.016 & 0.148 & -0.008 & 0.115 & -0.006 & 0.053 \\
& line & 0.004 & 0.144 & 0.000 & 0.112 & 0.003 & 0.046 \\
\hline \multirow{2}{*}{$10-11$} & sample & -0.006 & 0.145 & -0.005 & 0.136 & 0.003 & 0.056 \\
& line & -0.003 & 0.137 & -0.009 & 0.138 & 0.000 & 0.053 \\
\hline \multirow{2}{*}{$11-12$} & sample & 0.000 & 0.141 & 0.004 & 0.169 & 0.007 & 0.060 \\
& line & 0.005 & 0.147 & -0.022 & 0.178 & -0.003 & 0.058 \\
\hline \multirow{2}{*}{$12-13$} & sample & 0.011 & 0.152 & -0.020 & 0.213 & 0.001 & 0.068 \\
& line & -0.013 & 0.146 & -0.015 & 0.217 & -0.011 & 0.067 \\
\hline \multirow{2}{*}{$13-14$} & sample & 0.004 & 0.168 & -0.027 & 0.257 & -0.004 & 0.084 \\
& line & -0.010 & 0.158 & -0.044 & 0.261 & -0.014 & 0.078 \\
\hline \multirow{2}{*}{ overall } & sample & -0.000 & 0.151 & -0.012 & 0.192 & 0.000 & 0.065 \\
& line & -0.006 & 0.147 & -0.020 & 0.196 & -0.007 & 0.063 \\
\hline
\end{tabular}

magnitudes, zoomed for more visibility. Generally, stars with $>10$ magnitude are relatively weak and under-sampled.

Image distortion effects generally caused measured star positions to have larger systematic errors when located away from the centre of an image. So only those stars positioned in the central region of $724 \times 724$ pixels in each image were selected for measurement.

We measured these stars' centre positions in all images by using each of the three centroiding methods: modified moment, twodimensional Gaussian fitting and median, respectively. The computed position of every measured star in an image was derived by reducing its corresponding reference position from the Gaia DR2 catalogue to its CCD image coordinates. Finally, the observedminus-calculated (O-C) residuals between the measured position and the calculated position were evaluated.

After these processing steps, each algorithm obtained 3845 stars' measured positions and their corresponding (O-C)s. The mean and standard deviation of these (O-C)s for each method were then used to compare their accuracy and precision.

The (O-C)s of 3845 stars measured by these three centroiding algorithms are shown in Fig. 4. Each star's (O-C)s are displayed in sample and line along with the stars' magnitudes, respectively. The mean and standard deviations of 3845 (O-C)s by each method are shown in Table 1.

From Fig. 4, it can be found that the results of the three algorithms in sample (x) are similar to those in line (y). With the increase of the star magnitudes, each method's precision becomes worse, and this is most obvious in the median method. Fig. 4 also indicates that the modified moment method performs best. The median method is better than Gaussian fitting for smaller magnitudes, but worse than Gaussian fitting for greater magnitudes. Table 1 gives the statistical results of the three algorithms in different magnitude ranges, and overall magnitudes. It shows that the modified moment has the best precision in different magnitude ranges com- pared to the median and Gaussian fitting methods. In the overall magnitudes, its standard deviation is 0.065 pixels in sample and 0.063 pixels in line. The precision is over two times better than that of the Gaussian fitting, and three times better than that of the median.

In general, the Gaussian fitting is a very good method (van Altena \& Auer (1975), Li et al. (2009) ). As Stone (1989) points out, the median method is usually not considered to be satisfactory. However, in the astrometry of Cassini ISS images, the modified moment is better than the Gaussian fitting and median. The main reason for this is that point sources are under-sampled (their FWHMs are 1.3 pixels, and less than 2 pixels). This causes the intensity profile of a point source to be far away from the ideal Gaussian bell shape that is our usual assumption about a point source's intensity profile. Of course, this conclusion is specific to the Cassini ISS images studied here. For other space images, further evaluation should be performed.

\section{ASTROMETRIC REDUCTION OF ANTHE}

We downloaded all possible Cassini ISS images with Anthe within the field of view, taken between 2008 and 2017 from the PDS website (http: //pds-imaging.jpl .nasa.gov/). The total number of images was 5444. In most of them, Anthe was not detectable because of its faintness and small size. In the remaining images, Anthe is detectable as an unresolved point-like object, so centroiding methods could be used to measure its position. Some images were discarded due to noise pollution, while some WAC images were also discarded because Anthe's location was considered too far away from the centre of the image to be measured without excessive distortion. In total, 182 images of Anthe were measured successfully, including 112 out of 1,010 NAC images, and 70 out of 4,434 WAC images. All the measured images are taken with filters CL1 and CL2. Some typical ISS images of Anthe are shown in Fig. 5.

Anthe was measured by Caviar in which we added the option of the modified moment method, as an alternative to the built-in 2D Gaussian method. At present, in the modified Caviar, the user can choose the modified moment method or the Gaussian method to obtain the centre of a point-like object.

The astrometric reduction was divided into four steps: (1) camera pointing correction, (2) Anthe centre measurement and phase correction, (3) Anthe centre position reduction, (4) comparison with JPL's SAT393 ephemeris.

In the first step, the centroiding method was used to measure every imaged star's centre. These positions were then matched to positions from the Gaia DR2 catalogue to correct the camera's pointing. In the second step, the centroiding method was applied to measure Anthe's centre, and a correction made to Anthe's position for the solar phase angle. In the third step, Anthe's image coordinates were converted to right ascension and declination in the International Celestial Reference Frame (ICRF), with origin at Cassini's centre. In the fourth step, we converted Anthe's position provided from JPL's SAT393 ephemeris to its equivalent in the ICRF centered at $C$ assini, in image coordinates, and then computed the $(\mathrm{O}-\mathrm{C}) \mathrm{s}$. The details of the reduction can be found in Cooper et al. (2006)

In order to compare the modified moment method to the existing 2D Gaussian fitting in Caviar, we measured all 112 NAC images of Anthe twice according to the above 4 steps: once for each method. That is, firstly the Gaussian fitting was used in step 1 

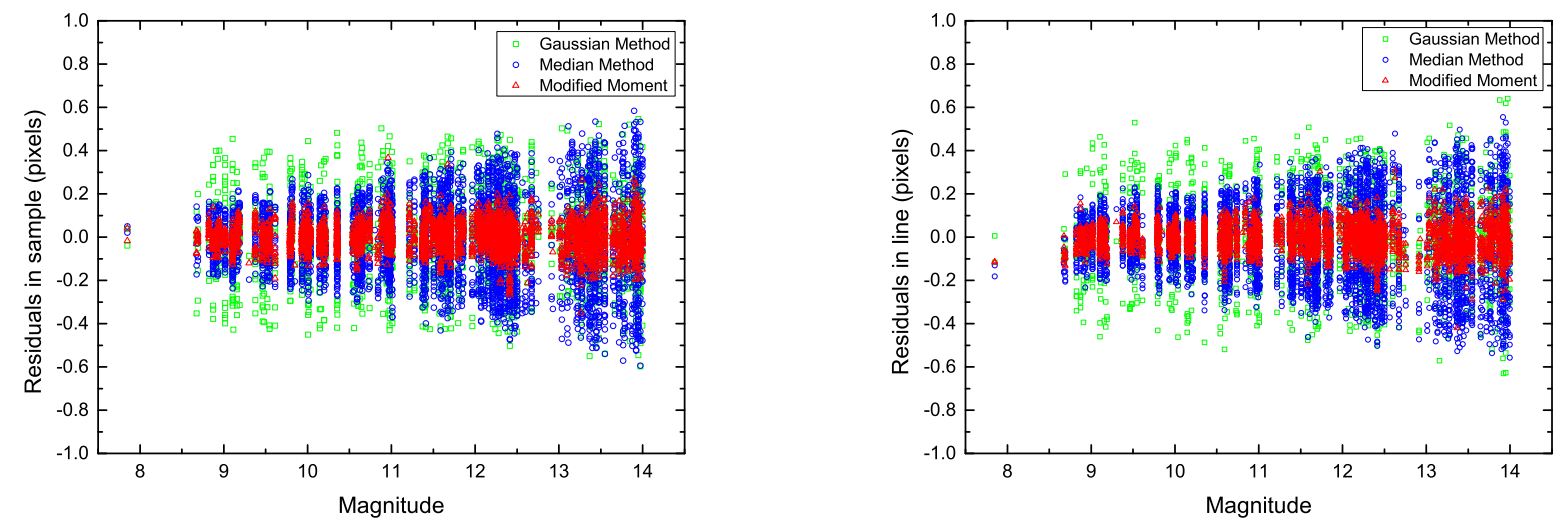

Figure 4. The residuals of all 3845 stars relative to Gaia DR2 in sample and line measured by two-dimensional Gaussian fitting, median method and modified moment method, respectively.
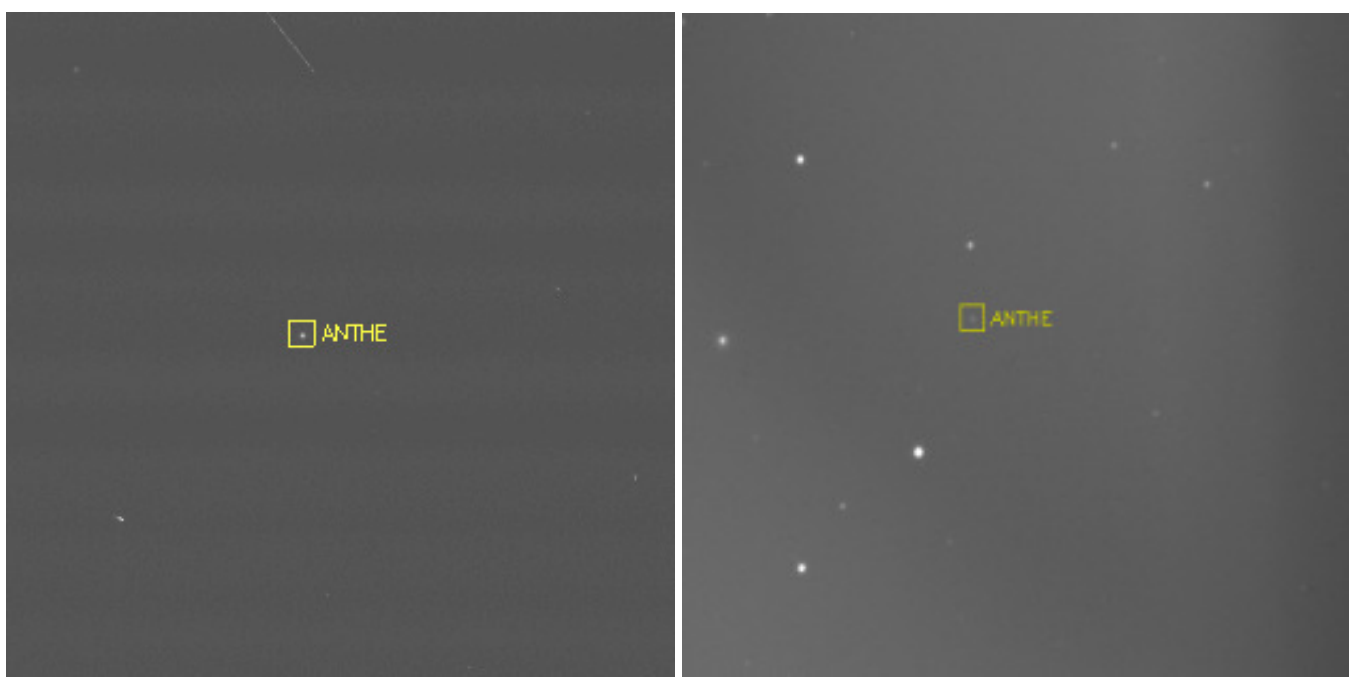

Figure 5. Some typical measurable images of Anthe. The left-hand figure is NAC image, and the right-hand figure is WAC image, Anthe is marked by a yellow box. Both of them are transformed for more visibility

and 2 to measure the imaged stars' positions and Anthe's positions. During the two steps of measurement, we used the same Guassian fitting in each step. Following this, the experiment was repeated except that the modified moment was used in step 1 and 2 . We then calculated the means and standard deviations of the (O-C)s of Anthe's positions in image coordinate obtained by the two different measurement methods. The final results are shown in Table 2.

Table 2 shows that the mean and standard deviation of the $(\mathrm{O}-$ C)s of the modified moment method are better than that of the 2D Gaussian fitting. It indicates that the modified moment still keeps the advantages over Gaussian fitting in the measurement of a pointlike satellite. Because some images of Anthe have a slightly larger apparent diameter than the undersampled stars, the advantage of the modified moments slightly decreased. Furthermore, the error in the computation of the phase effect is more visible for such images, which also decreases the precision of the measurement.

Following these steps, we used the modified moment method to measure all 182 images of Anthe (112 NAC and 70 WAC images). As we know, the solar phase angle (observer-object-Sun) will bring about bias on the positional measurement of planets and nat-
Table 2. Mean values (mean) and standard deviations (std) of the (O-C)s of all Anthe NAC images by using two-dimensional Gaussian fitting and modified moment (in pixels).

\begin{tabular}{|c|c|c|c|c|}
\hline \multirow{2}{*}{ methods } & \multicolumn{2}{|c|}{ Gaussian } & \multicolumn{2}{c|}{ Modified Moment } \\
\cline { 2 - 5 } coordinates & mean & std & mean & std \\
\hline sample & -0.018 & 0.166 & -0.013 & 0.082 \\
\hline line & 0.040 & 0.160 & 0.009 & 0.103 \\
\hline
\end{tabular}

ural satellites in the solar system. In ISS observations, Cooper et al. (2006) has reported the offset of the centroid of Amalthea caused by the phase angle can reach -0.491 pixels in line and 0.12 pixels in sample. So the phase effect may be significant in ISS observations. We made the phase correction for all 182 Anthe centre positions. The bias caused by the phase angle was calculated following the principle and equations described in Cooper et al. (2006), Lindegren L. (1977), and Hestroffer D. (1998). In the procedure, we assume that Anthe is a sphere and its surface obeys Lambert's law of scattering light. In all 182 Anthe ISS observations, the phase an- 
Table 3. Mean values (mean) and standard deviations (std) of the measurements of all Anthe ISS NAC/WAC images by modified moment method.

\begin{tabular}{c|c|c|c|c}
\hline \multirow{2}{*}{ direction } & \multicolumn{2}{|c|}{ NAC } & \multicolumn{2}{c}{ WAC } \\
\cline { 2 - 5 } & mean & std & mean & std \\
\hline sample(pixel) & -0.013 & 0.082 & 0.020 & 0.086 \\
line(pixel) & 0.009 & 0.103 & 0.012 & 0.046 \\
$\alpha^{*} \cos \delta(")$ & -0.052 & 0.102 & -0.709 & 0.907 \\
$\delta(")$ & 0.012 & 0.117 & 0.020 & 0.727 \\
$\alpha^{*} \cos \delta(\mathrm{km})$ & -0.284 & 0.696 & -3.265 & 3.581 \\
$\delta(\mathrm{km})$ & 0.022 & 0.917 & -0.008 & 4.017 \\
\hline
\end{tabular}

gles range from 9.66 to 107.59 degrees. The maximum phase error in image coordinates reached 0.068 pixels.

In Fig. 6 and 7, we show the (O-C) residuals relative to the SAT393 ephemeris of 112 NAC images and 70 WAC images. Fig. 6 shows the residuals in sample and line, for each observation of Anthe, in pixels. Fig. 7 shows the distance residuals in $\alpha \times \cos (\delta)$ and $\delta$ directions in kilometres. The statistical results of these residuals are given in Table 3 .

It can be seen that the mean values of the NAC positions of Anthe are close to zero, and the standard deviations are 0.082 and 0.103 pixels in sample and line, respectively. In terms of angle, the standard deviations of residuals are $0.102^{\prime \prime}$ in $\alpha \times \cos (\delta)$ direction and $0.117^{\prime \prime}$ in $\delta$ direction; from the view of distance, the standard deviations of residuals are 0.696 and $0.917 \mathrm{~km}$, respectively. Anthe's WAC positions show similar results. Due to the lower resolution of WAC compared to the NAC, its residuals are better in image coordinates, and worse in angle and distance in right ascension and declination in the ICRF.

Table 4 gives a sample of the complete set of reduced Anthe observations. It includes the ISS image name, observation midtime, measured position (sample and line), the equivalent right ascension and declination in the ICRF centered Cassini, measured pointing information, and phase biases. Column 1 is the image name. Column 2 is the date and exposure mid-time of the image (UTC). The columns $\alpha_{c}, \delta_{c}$, and Twist refer to the right ascension, declination, and twist angle of the camera's pointing vector in the ICRF centered Cassini. The columns of sample and line are the observed position with phase correction of Anthe in the image, its corresponding right ascension and declination in the ICRF are shown in columns $\alpha$ and $\delta$. Columns BiasX and BiasY are the offsets of Anthe in sample and line caused by the phase effect. It should be noted that the columns of sample and line are the measured positions with phase correction, not the photocentres (raw measured positions) of Anthe. The photocentre of Anthe can be derived by the columns of sample and line minus the columns BiasX and Bias Y, respectively. Furthermore, users can modify the measured positions and phase biases by their better model of phase correction, if so desired.

\section{CONCLUSIONS}

Generally, Gaussian centroiding can achieve good accuracy and precision. But in Cassini ISS images, the objects (point-like stars and/or satellites) are often poorly resolved into pixels, and most images are dark. The intensity distribution of an object's image is often far away from a Gaussian function, so that the performance of Gaussian centroiding is decreased significantly. Median centroiding is better than Gaussian centroiding for bright stars. However, its performance degrades as the magnitude of an object increases. With modified moment centroiding, we propose an automatic back- ground determination scheme that reduces greatly the influence of sky background. So the modified moment achieves the best accuracy and precision of the three methods.

From our comparative experiment of 56 Cassini ISS images of sky, we conclude that the modified moment performs the best of the three centroiding methods: modified moment, median and Gaussian fitting for the positional measurements of stars. It can reach a standard deviation of 0.065 pixels and 0.063 pixels in sample and line, respectively. This precision is better than the existing Gaussian fitting in Caviar by over a factor of 2 . The pointing correction in astrometry of ISS images will profit from applying the modified moment centroiding on the positional measurements of stars.

From the comparative experiment of 112 NAC images of Anthe, we also conclude that the modified moment method is better than the existing Gaussian fitting in Caviar for the positional measurement of the point-like satellites. Due to the error arising from the computation of the phase effect, and changes in the apparent size of Anthe, the precision of the modified moment decreased slightly.

Finally, we used the modified moment method to measure Anthe's position from Cassini ISS NAC and WAC images taken from 2008 to 2017. A total of 182 available measurements were obtained. Compared to JPL's SAT393 ephemeris, the average residuals of all NAC images in right ascension and declination directions were -0.284 and $0.022 \mathrm{~km}$, and the standard deviations were 0.696 and $0.917 \mathrm{~km}$, respectively. For WAC images, the mean values of all residuals were -3.265 and $-0.008 \mathrm{~km}$, and the standard deviations were 3.581 and $4.017 \mathrm{~km}$, respectively.

In the future, we will add the modified moment method into the next release of Caviar. Furthermore, according to this study, Cassini ISS astrometry of unresolved objects, such as small moons, can benefit from using the modified moment method in their astrometric reduction. Consequently, we intend to extend this work to re-reduce Cassini ISS astrometric data for moons other than Anthe, in order to take full advantage of the potential improvements in precision possible using this new method.

\section{ACKNOWLEDGEMENTS}

This work was partly supported by National Natural Science Foundation of China (Grant Nos.U2031104, 62071201 and 11873026). This work has also been supported by the European Community's Seventh Framework Program (FP7/2007-2013) under grant agreement 263466 for the FP7-ESPaCE project and the International Space Science Institute (ISSI).

\section{DATA AVAILABILITY}

The full version of Table 4 is only available at the CDS via anonymous ftp to cdsarc.u-strasbg.fr (130.79.128.5) or via https://cdsarc.u-strasbg.fr/ftp/vizier.submit//A_Comparison_of _Centering_in_ISS_Astrometry/. The routine for the modified moment algorithm will also be shared on request to the corresponding authors.

\section{References}

Anderson J., King I. R. 2000. PASP, 112, 1360

Cooper N. J., Murray C. D., Porco C. C., \& Spitale J. N. 2006, Icarus, 181, 223 

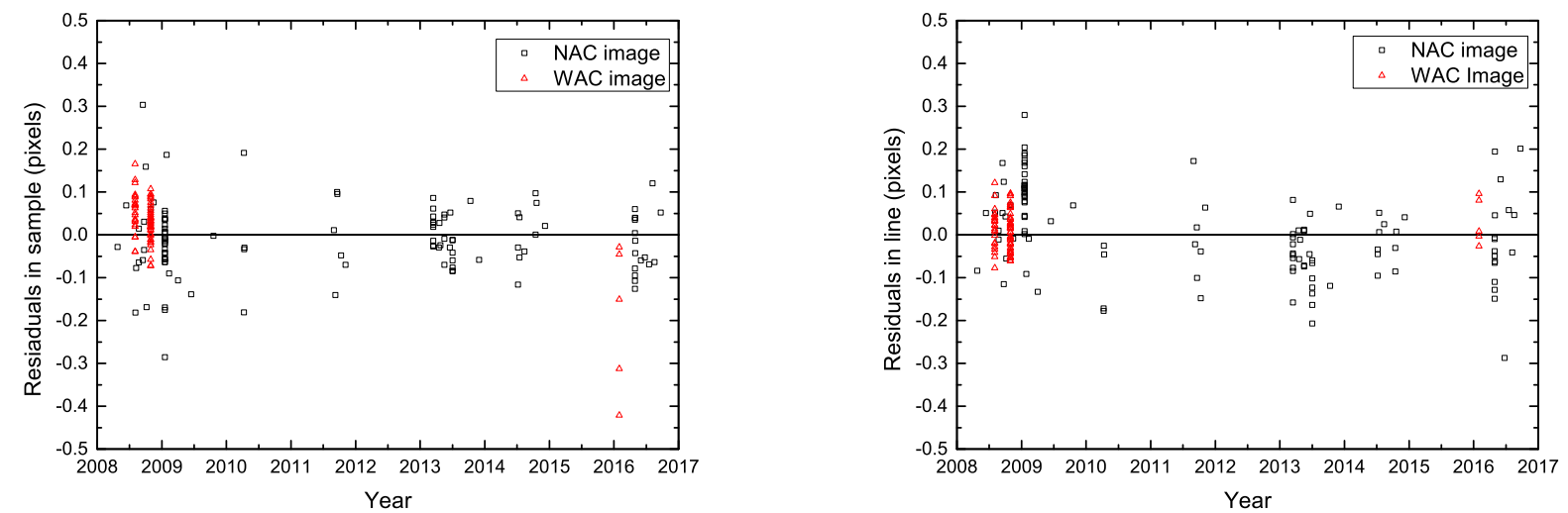

Figure 6. The (O-C)s of Anthe's NAC and WAC observations relative to the JPL Sat393 ephemeris in sample (x) and line (y), respectively, in pixels.
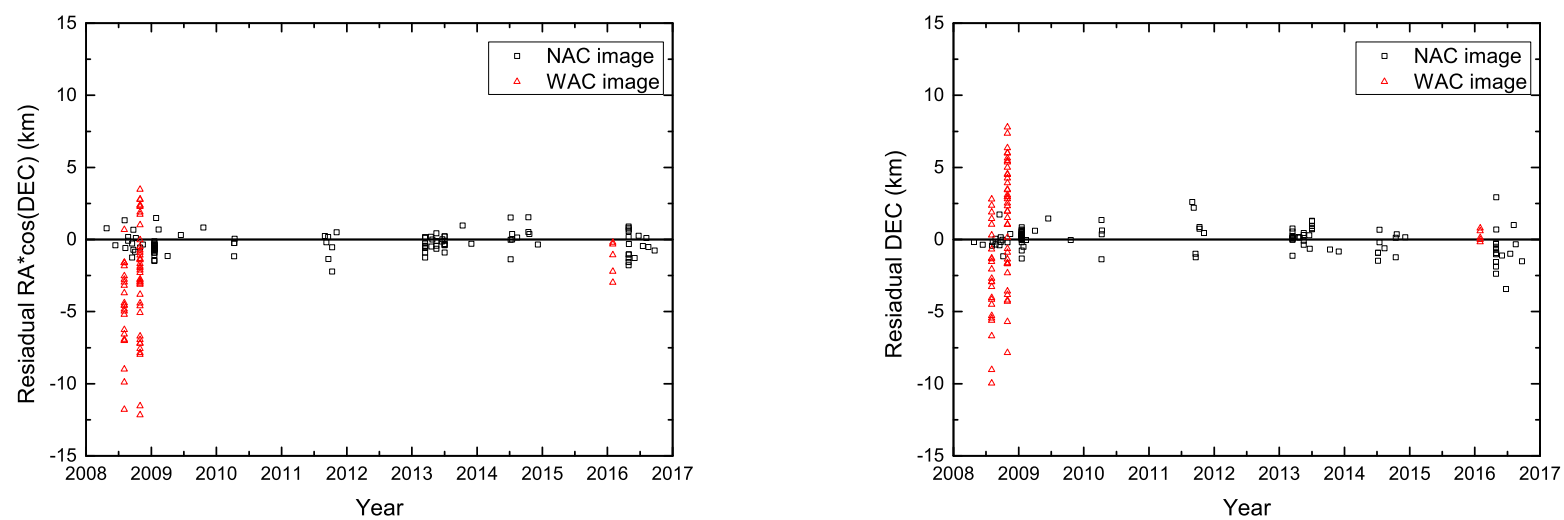

Figure 7. The (O-C)s of Anthe's NAC and WAC observations relative to the JPL Sat393 ephemeris in $\alpha^{*} \cos \delta$ and $\delta$, respectively, in kilometres.

Table 4. A sample of Cassini ISS observations of Anthe. Column 1 is the image name, Column 2 is the date and exposure mid-time of the image (UTC), the columns $\alpha_{c}, \delta_{c}$, and Twist refer to the right ascension, declination, and twist angle of the camera's pointing vector in the ICRF centered Cassini, while columns $\alpha$ and $\delta$ are the right ascension and declination in the ICRF for Anthe. The columns of sample and line are the measured position of Anthe with phase correction. The origin of the sample, line coordinate system is at the top left of the image, and line y increasing downwards and sample $\mathrm{x}$ to the right. the last two columns are the phase errors in sample and line. All the angle variables are given in degrees. Image size is 1024 by 1024 pixels. The full table is availalbe online.

\begin{tabular}{|c|c|c|c|c|c|c|c|c|c|c|}
\hline Image ID & Mid-time (UTC) & $\begin{array}{r}\alpha_{c} \\
(\mathrm{deg})\end{array}$ & $\begin{array}{r}\delta_{c} \\
(\mathrm{deg})\end{array}$ & $\begin{array}{l}\text { Twist } \\
\text { (deg) }\end{array}$ & $\begin{array}{r}\text { Sample } \\
(\mathrm{px})\end{array}$ & $\begin{array}{r}\text { Line } \\
(\mathrm{px})\end{array}$ & $\begin{array}{r}\alpha \\
(\mathrm{deg})\end{array}$ & $\begin{array}{r}\delta \\
(\operatorname{deg})\end{array}$ & $\begin{array}{r}\text { BiasX } \\
(\mathrm{px})\end{array}$ & $\begin{array}{r}\text { BiasY } \\
(\mathrm{px})\end{array}$ \\
\hline N1587716907 & 2008 APR 24 07:51:09.525 & 146.596805 & -8.029986 & 96.388351 & 541.13 & 546.31 & 146.583658 & -8.021207 & 0.0051 & -0.0045 \\
\hline N1591878477 & 2008 JUN 11 11:50:10.123 & 152.981812 & 4.587787 & 186.063310 & 518.05 & 487.79 & 152.978694 & 4.595645 & -0.0015 & -0.0008 \\
\hline N1596338308 & 2008 AUG 02 02:40:09.689 & 161.855395 & -19.615422 & 178.070802 & 524.21 & 513.32 & 161.850734 & -19.615900 & 0.0000 & -0.0142 \\
\hline N1596721036 & 2008 AUG 06 12:58:54.984 & 142.744678 & 18.739383 & 66.938426 & 537.76 & 505.02 & 142.750556 & 18.746808 & 0.0000 & -0.0111 \\
\hline W1832830863 & 2016 JAN 30 06:36:45.689 & 288.561582 & 9.192775 & 358.298330 & 491.81 & 521.53 & 288.494324 & 9.229108 & -0.0433 & -0.0339 \\
\hline W1832830969 & 2016 JAN 30 06:38:31.876 & 289.205222 & 9.169032 & 358.274785 & 491.71 & 521.62 & 289.137651 & 9.205691 & -0.0447 & -0.0345 \\
\hline W1832831075 & 2016 JAN 30 06:40:16.926 & 289.844198 & 9.143690 & 358.251696 & 491.60 & 521.65 & 289.776270 & 9.180507 & -0.0462 & -0.0351 \\
\hline
\end{tabular}

Cooper N. J., Murray C. D., Beurle K., et al. 2008, Icarus, 195, 765

Cooper N. J., Murray C. D., Lainey V., Tajeddine R., Evans M. W., \& Williams G. A. 2014, A\&A, 572, A43

Cooper N. J., Lainey V., Meunier L. E., et al. 2018. A\&A, 610, A2

Delabie J., Schutter J. D., Vandenbussche B. 2014. J of Astronaut Sci, 61, 60

Gaia Collaboration, Brown A. G. A., Vallenari A., et al., 2018, A\&A, 616, A1
Gaia Collaboration, 2018, yCat, I/345

Hedman, M.M., Murray, C.D., Cooper, N.J., Tiscareno, M.S., Beurle, K.,

Evans, M.W., Burns, J.A., 2008, Icarus, 199, 378

Hestroffer D., 1998, A\&A, 336, 776

Lainey V., Casajus L. G., Fuller J., et al., 2020, NatAs, 4, 1053

Li Z., Peng Q. Y., Han G. Q., 2009, AcASn, 50, 340

Lindegren L., 1977, A\&A, 57, 55

Lu T., Luo W., Zhang J., et al., 2018, AJ, 156, 14 
Munoz-Gutierrez M. A., Winter S. G., 2017. MNRAS, 470, 3750

Madeira G., Winter S. M. G., 2020, Eur. Phys. J. ST, Special topics 229, 1527

Porco C. C., West R. A, Squyres S., et al., 2004, SSRv, 115, 363

Porco, C.C., \& the Cassini Imaging Team, 2007, IAUC. 8557

Sun K. L., Seiß M., Hedman M. M., Spahn F., 2017, Icar, 284, 206

Sun R. Y., Zhao C. Y., 2014, AdSpR, 53, 1664

Stetson P. B., 1987. PASP, 99, 191

Stone R. C., 1989. Astron. J., 97, 1227

Tajeddine R., Cooper N. J., Lainey V., Charnoz S., \& Murray C. D., 2013, A\&A, 551, A129

Tajeddine R., Lainey V., Cooper N. J., \& Murray C. D., 2015, A\&A, 575, A73

van Altena W. F., Auer L. H., 1975, ASSL, 411.

Wang H., Xu E., Li Z., Li J., Qin T., 2015, AdSpR, 56, 2196

Zhang Q. F., Lainey V., Cooper N. J., Vienne A., Peng Q. Y., \& Xiong Y. T., 2018, MNRAS, 481, 1, 98

This paper has been typeset from a $\mathrm{T}_{\mathrm{E}} \mathrm{X} / \mathrm{L} \mathrm{AT} \mathrm{EX}$ file prepared by the author. 